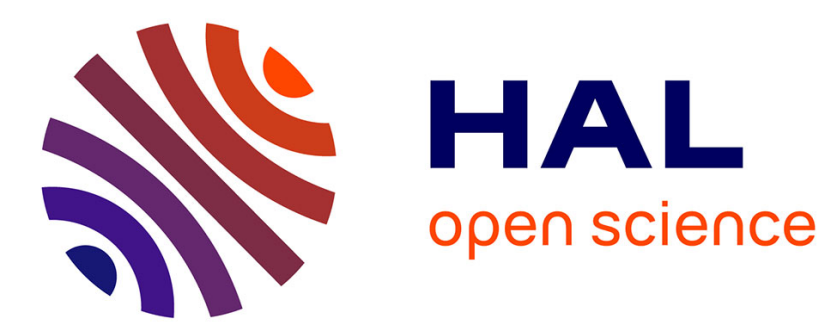

\title{
Practical laws for natural convection of viscous fluids heated from above in a shallow cavity \\ Franck Pigeonneau, Jean-Marc Flesselles
}

\section{To cite this version:}

Franck Pigeonneau, Jean-Marc Flesselles. Practical laws for natural convection of viscous fluids heated from above in a shallow cavity. International Journal of Heat and Mass Transfer, 2012, 55 (1-3), pp.436-442. 10.1016/j.ijheatmasstransfer.2011.09.042 . hal-00648070

\section{HAL Id: hal-00648070 \\ https://hal.science/hal-00648070}

Submitted on 5 Dec 2011

HAL is a multi-disciplinary open access archive for the deposit and dissemination of scientific research documents, whether they are published or not. The documents may come from teaching and research institutions in France or abroad, or from public or private research centers.
L'archive ouverte pluridisciplinaire HAL, est destinée au dépôt et à la diffusion de documents scientifiques de niveau recherche, publiés ou non, émanant des établissements d'enseignement et de recherche français ou étrangers, des laboratoires publics ou privés. 


\title{
Practical laws for natural convection of viscous fluids heated from above in a shallow cavity
}

\author{
F. Pigeonneau ${ }^{*, a}$, J.-M. Flesselles ${ }^{\mathrm{b}}$ \\ ${ }^{a}$ Surface du Verre et Interfaces, UMR 125 CNRS/Saint-Gobain, 39 quai Lucien Lefranc - BP 135, \\ 93303 Aubervilliers cedex France \\ ${ }^{b}$ Saint-Gobain Recherche, 39 quai Lucien Lefranc - BP 135, 93303 Aubervilliers cedex France
}

\begin{abstract}
Industrial production of glass involves natural convection of viscous fluids heated from above in shallow cavities. This problem is revisited by means of extensive numerical simulations and analytical expansions for a 2D configuration.

This approach yields exact and approximate analytical expressions that can be practically used to evaluate the convective intensity and the heat flux in the different regimes.

These results also confirm that the control parameter for this problem is the product of the Rayleigh number, based on the height of the enclosure, by the cavity aspect ratio squared whatever the top boundary conditions.
\end{abstract}

Key words: Natural convection, glass furnace, Nusselt number, shallow cavity, asymptotic analysis, numerical simulation

\section{Nomenclature}

Roman symbols

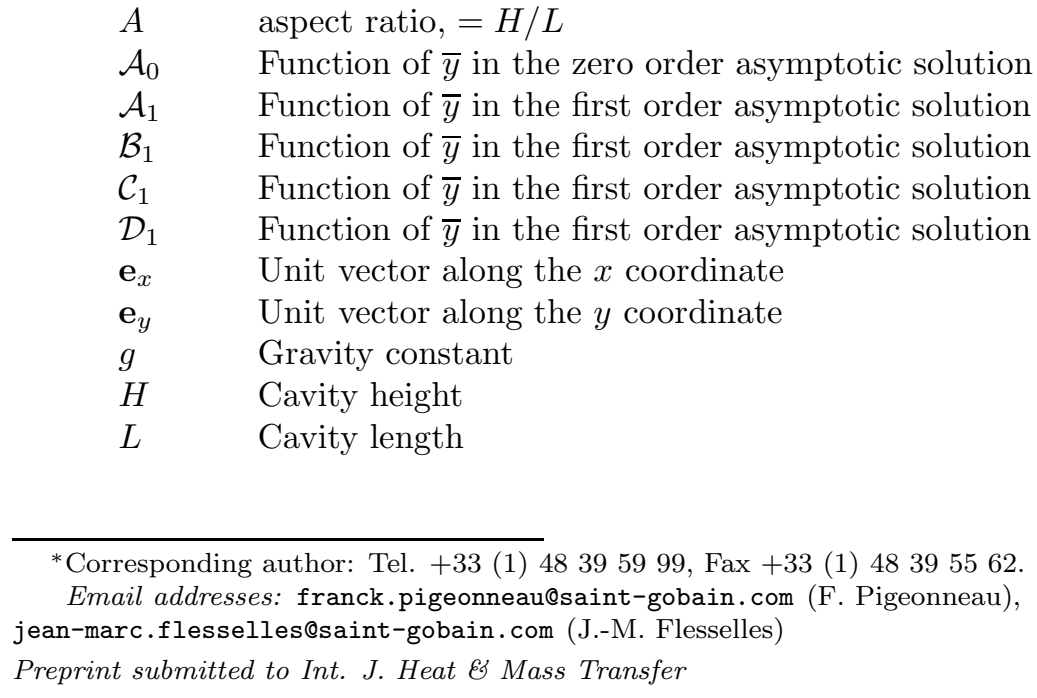

\footnotetext{
*Corresponding author: Tel. +33 (1) 483959 99, Fax +33 (1) 48395562 . Email addresses: franck.pigeonneau@saint-gobain.com (F. Pigeonneau), jean-marc.flesselles@saint-gobain.com (J.-M. Flesselles)

-
-
-
-
-
-
-
-
$\mathrm{m} \cdot \mathrm{s}^{-2}$
$\mathrm{~m}$
$\mathrm{~m}$


$\mathrm{Nu} \quad$ Nusselt number, $=(\partial T / \partial n) /(\Delta T / H)$

$\langle N u\rangle \quad$ Average Nusselt number based on $L_{2}$ norm of $N u$

$P, \bar{P} \quad$ Dimensionless pressure

$P e \quad$ Péclet number, $=u_{0} L / \kappa$

$\operatorname{Pr} \quad$ Prandtl number, $=\nu / \kappa$

$\mathrm{Ra} \quad$ Rayleigh number based on height $H$

$T \quad$ Temperature

$u, \bar{u}$

Dimensionless

$v, \bar{v}$

Dimensionless vertical velocity component
Dimensionless longitudinal coordinate

$x, \bar{x}$

$y, \bar{y} \quad$ Dimensionless vertical coordinate

Greek symbols

$\begin{array}{lll}\beta & \text { Heat expansion coefficient } & \mathrm{K}^{-1} \\ \Delta T & \text { Temperature difference, }=T_{+}-T_{-} & \mathrm{K} \\ \kappa & \text { Heat diffusivity } & \mathrm{m}^{2} \cdot \mathrm{s}^{-1} \\ \nu & \text { Kinematic viscosity } & \mathrm{m}^{2} \cdot \mathrm{s}^{-1} \\ \theta & \text { Dimensionless temperature, }=\left(T-T_{-}\right) /\left(T_{+}-T_{-}\right) & -\end{array}$

Subscripts

$0 \quad$ Characteristic scale or zeroth order asymptotic solution

$1 \quad$ First order asymptotic solution

$+\quad$ Cold temperature

- $\quad$ Hot temperature

Superscripts

1 Derivate with respect to $\bar{y}$

\section{Introduction}

Although generally poorly known to the general public, industrial glassmaking involves multiple heat and mass transfer phenomena. Glass furnaces are essentially made up of a 
combustion system and a tank within which raw materials undergo a series of high temperature chemical reactions, finally producing a homogeneous glass. Within these long and shallow tanks, convective motion driven by controlled temperature gradients move the liquid "uphill". The magnitude of the velocity sets the residence time distribution and the mixing intensity, both of which impact the power needed to make glass, i.e. the eventual energy consumption of an industrial plant. Although velocity and temperature fields in glass furnaces can be computed using various numerical tools, the understanding of heat and mass transfer phenomena is still partial. Furnace design should be improved through better knowledge of the key parameters driving natural convection inside the glass bath.

The purpose of this paper is to give a physical insight to the phenomena at play and provide analytical "ready to use" formula on natural convection.

At high temperature, molten glass is a Newtonian fluid : its mechanical properties are properly described by a scalar viscosity, that, depends on temperature [16]. In large window glass furnaces, where the melt temperature varies from 1200 to $1500{ }^{\circ} \mathrm{C}$, viscosity ranges over about one order of magnitude. Molten glass being also a semi-transparent media, heat transfer is not only conductive but also radiative. However, for most common glasses, including ordinary transparent window glass, the absorption length is such that radiation can be approximated by a conductive model $[9,17]$ where the equivalent heat conductivity varies like $T^{3}$ (Rosseland approximation). As a consequence the Prandtl number based on the effective conductivity and the viscosity ranges from $2 \cdot 10^{3}$ to $2 \cdot 10^{4}$. Detailed calculations outside the scope of the paper show that the actual impact of these variations does not bring any new phenomena.

Since furnaces are generally longer and wider than they are high (for a precise description of technology see Ref. [19]), main flow structures take place in the longitudinal plane. The problem can thus be studied in a long $2 \mathrm{D}$ cavity. To mimic the fact that glass is heated by gas flames from above and cooled by raw materials floating on the surface next to where they are charged, an uneven temperature profile is applied on the upper boundary. The presence of raw materials and sometime foam floating at the surface of a glass furnace make it difficult to describe the kinematic conditions for the liquid. It is known in this kind of convection problems [4] that the choice of slip or no slip does not modify the fluid physics at play in the bulk nor the scaling laws for the overall motion. For our goal, we do choose the simplest case for our model, i.e. an all no-slip condition enclosure. Although a number of authors have recently studied similar problems $[4,10]$, none has yet provided a set of laws that could be used by a practitioner to design such a convection tank and control the flow structure.

In the following, a sinusoidal temperature profile is imposed along the top of the cavity like already done by Somerville [18]. Such a choice brings numerous benefits: it allows for a regular solution at the corners and makes some analytical calculations possible. That does not restrict the generality of the analysis since it is the first term of a Fourier development of any similar, realistic, imposed temperature profile. Finally, in the following analysis, the Prandtl number is assumed to be large in order to mimic glass properties.

This situation is not far from the case where the cavity is heated along its side walls. In both cases, convection occurs without threshold and is mainly driven by the longitudinal thermal gradient. As it has been shown in [7], scaling laws can be obtained by balancing terms in the equations of motions, without involving the actual boundary conditions. For 
the cavity heated along its side walls, Böhrer [3] compiled a large amount of numerical and experimental data. He studied kinematic and thermal structures and suggested that the governing parameter is the product of the Rayleigh number by the aspect ratio squared ${ }^{1}$, $R a A^{2}$. Later, Flesselles and Pigeonneau [7] presented a scale analysis to explain why $\mathrm{Ra} A^{2}$ is the governing parameter. That is compatible with the results of Chiu-Webster et al. [4], where a linear profile was applied to the upper limit, but analyzed with a different set of variables (although with similar notations).

The problem is presented in section 2, where cavity geometry and boundary conditions are defined. Section 3 is devoted to the presentation of the numerical results and their analysis. Conclusions are given in section 4. Appendix A details the asymptotic solution of heat and mass transfer in the enclosure when the aspect ratio is small.

\section{Problem statement}

We consider a two-dimensional rectangular shallow cavity of height $H$ and length $L$ placed within the gravity field $g$. It is filled with an incompressible fluid, the kinematic viscosity, $\nu$, and the heat diffusivity, $\kappa$, of which are constant. Figure 1 gives the kinematic and thermal boundary conditions. The following temperature profile is imposed along the upper boundary

$$
T(x)=T_{-}+\frac{\Delta T}{2}\left[1-\cos \left(\pi \frac{x}{L}\right)\right] .
$$

The temperature difference is defined by $\Delta T=T_{+}-T_{-}$where $T_{+}>T_{-}$. Adiabatic conditions are applied along the other walls. Equation (1) ensures continuity with the vanishing heat flux along the left and right sides. No-slip conditions are used along all boundaries.

The problem is governed by three dimensionless numbers (see Batchelor [1]) viz. the Prandtl number $P r$, the aspect ratio $A$, and the Rayleigh number $R a$ given respectively by:

$$
\begin{aligned}
\operatorname{Pr} & =\frac{\nu}{\kappa}, \\
A & =\frac{H}{L}, \\
R a & =\frac{g \beta \Delta T H^{3}}{\nu \kappa},
\end{aligned}
$$

where, in Eq. (2c), $\beta$ is the heat expansion coefficient. The Prandtl number $\operatorname{Pr}$ depends only on the fluid's physical properties. Previous work of Rossby [15], Lim et al. [13] and Gramberg et al. [10] has shown that heat and mass transfer are insensitive to the Prandtl number if the latter is large enough. Consequently, $\operatorname{Pr}$ is henceforth taken as constant and equal to $10^{3}$. In the following, the aspect ratio $A$ is less than unity. The Rayleigh number, $R a$, controls heat and mass transfer.

In articles [5, 7], the authors showed that the longitudinal velocity scales as $R a \kappa / L$ for small aspect ratios and small Rayleigh numbers. This velocity is used to normalize balance equations. All spatial coordinates are normalized by the height, $H$. Dimensionless equations written within the framework of the Oberbeek-Boussinesq approximation are

\footnotetext{
${ }^{1}$ Definitions of these quantities will be given below and can also be found in the Nomenclature.
} 

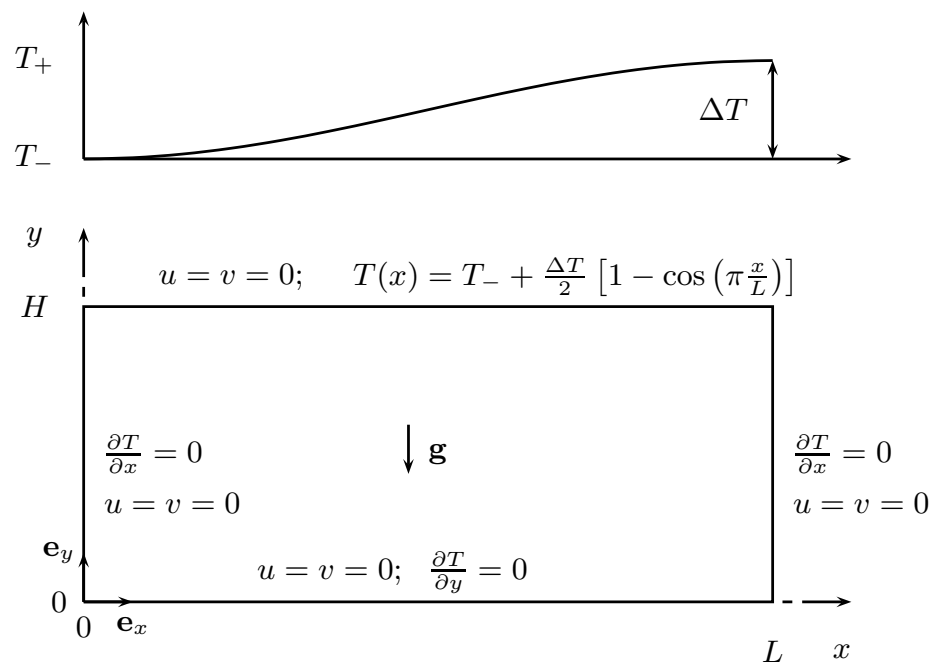

Figure 1: Rectangular cavity with kinematic and thermal boundary conditions.

$$
\begin{aligned}
\frac{\partial u}{\partial x}+\frac{\partial v}{\partial y} & =0 \\
u \frac{\partial u}{\partial x}+v \frac{\partial u}{\partial y} & =-\frac{\partial P}{\partial x}+\frac{\operatorname{Pr}}{\operatorname{RaA}}\left(\frac{\partial^{2} u}{\partial x^{2}}+\frac{\partial^{2} u}{\partial y^{2}}\right) \\
u \frac{\partial v}{\partial x}+v \frac{\partial v}{\partial y} & =-\frac{\partial P}{\partial y}+\frac{\operatorname{Pr}}{\operatorname{RaA}}\left(\frac{\partial^{2} v}{\partial x^{2}}+\frac{\partial^{2} v}{\partial y^{2}}\right)+\frac{\operatorname{Pr}}{R a A^{2}} \theta \\
u \frac{\partial \theta}{\partial x}+v \frac{\partial \theta}{\partial y} & =\frac{1}{R a A}\left(\frac{\partial^{2} \theta}{\partial x^{2}}+\frac{\partial^{2} \theta}{\partial y^{2}}\right) .
\end{aligned}
$$

The horizontal and vertical velocity components are, respectively, $u$ and $v$ where $P$ is the pressure. The dimensionless temperature is

$$
\theta=\frac{T-T_{-}}{T_{+}-T_{-}}
$$

We present herewithin a numerical solution obtained using a commercial CFD software based on the finite volume method. A segregated solver is used to solve iteratively each equation. Pressure and velocity fields are decoupled via the semi-implicit method for pressure linked equation (SIMPLE), [8]. The convective term gradients are determined by a third-order QUICK scheme introduced by Leonard [12]. About hundred cases have been run for which $A$ varies between $1 / 50$ and $1 / 5$ and $R a$ from 1 to $10^{9}$. The domain is meshed with a mapped grid using a square finite volume. The mesh size is equal to $2 \cdot 10^{-2}$ regardless of the Rayleigh number, so that the largest number of finite volumes is 125000 obtained for $A$ equal to $1 / 50$. This spatial resolution is enough to capture the 

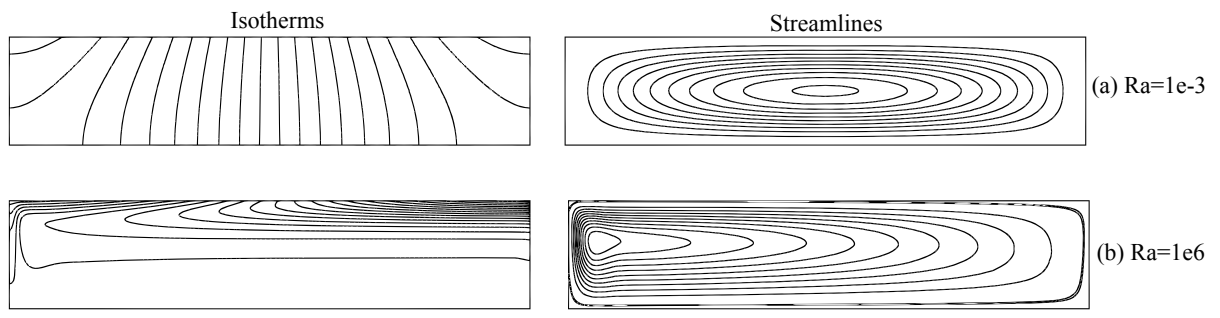

Figure 2: Isotherms and streamlines in a cavity with aspect ratio equal to $1 / 5$ for $R a=10^{-3}$ (first row) and $R a=10^{6}$ (second row).

boundary layer that is observed in the numerical solution and that follows the scaling established in the next section. The steady state solution is obtained when the residuals on the continuity and energy equations are equal to $10^{-8}$. For the momentum equations, the residuals are $10^{-6}$.

\section{Results}

Previous results $[3,4,7]$ suggest that two kinds of flow emerge. The first one, observed at small Rayleigh numbers, is dominated by thermal conduction and is called the conductive regime. The second kind of flow is obtained at large Rayleigh numbers and is characterized by a boundary layer below the upper boundary. This second regime is driven by convection and is therefore called the convective regime.

The typical thermal and kinematic patterns for (a) a small Rayleigh number, $\left(10^{-3}\right)$, and (b) a large $R a,\left(10^{6}\right)$, in a cavity with $A=1 / 5$ are shown in Figure 2. When $R a=10^{-3}$, the flow is in the conductive regime. The main thermal gradient is along the longitudinal coordinate. The streamlines present a pointwise symmetry around the center of the cavity. In the convective regime (see Figure 2-b), the symmetry is broken both on isotherms and streamlines. The thermal field is mainly driven by convection, which leads to the boundary layer just below the top boundary and a strong stratification in the bulk of the cavity. As seen in the streamline pattern, the flow is shifted toward the cold side (to the left of the enclosure). Close to the top left corner, the fluid falls down in the form of a plume.

In the following, the convective intensity and the heat flux on the upper limit are successively studied. The numerical results are compared to the asymptotic solution, given in Appendix A, obtained when $A$ and $R a$ are small.

\subsection{Péclet number in the enclosure}

The maximum of the $x$-velocity sets the velocity scale in the enclosure and characterizes the convective intensity. The Péclet number is defined as

$$
P e=\frac{|u|_{\max } L}{\kappa},
$$

where $|u|_{\max }$ is the maximum of the longitudinal velocity in physical dimensions. With the normalization used to numerically solve the problem, the $x$-velocity maximum is equal to the ratio $\mathrm{Pe} / \mathrm{Ra}$. 


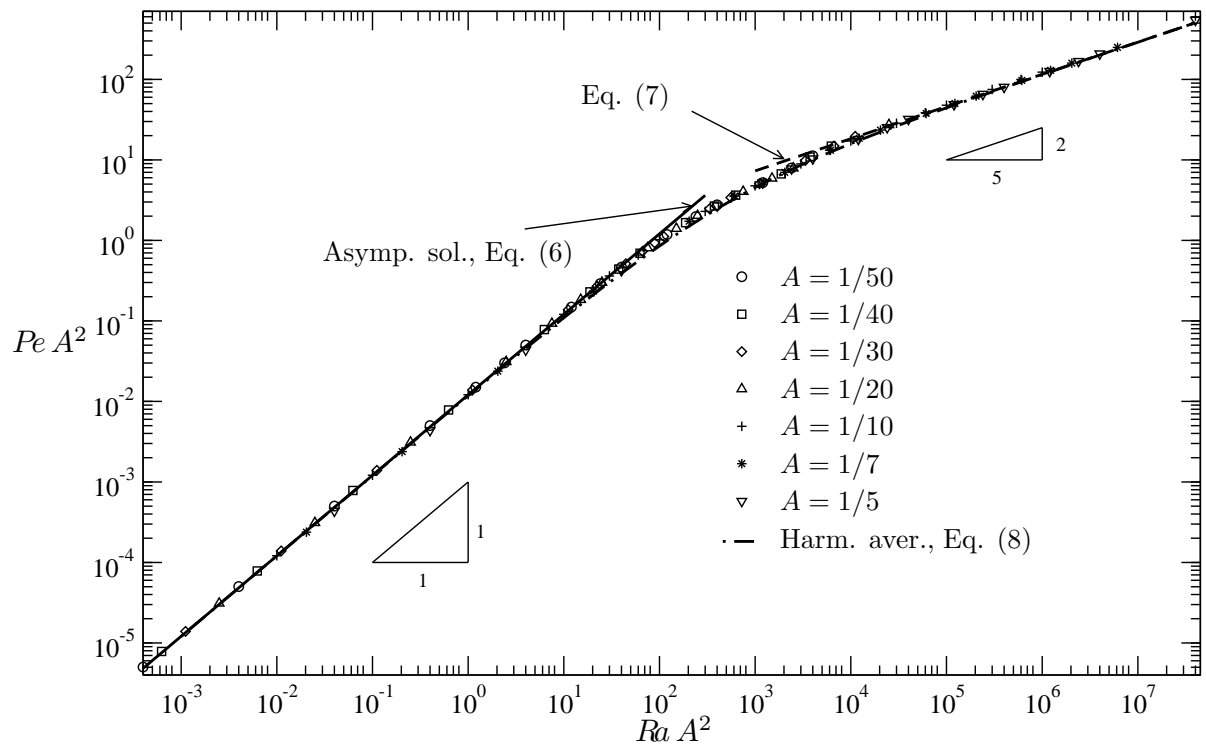

Figure 3: $P e A^{2}$ as a function of $R a A^{2}$ for cavities with aspect ratios of $1 / 50,1 / 40,1 / 30,1 / 20,1 / 10$, $1 / 7$ and $1 / 5$.

In order to investigate the convective intensity, $P e A^{2}$ versus $R a A^{2}$ is plotted in Figure 3 for seven aspect ratios and $R a$ ranging from 1 to $10^{9}$. The merging of all numerical data onto a single curve confirms that $R a A^{2}$ remains the control parameter whatever the boundary conditions: differentially heated end walls $[3,7]$ or an uneven top temperature profile. In both situations, convection occurs without threshold. It follows from the criteria pointed out by Joseph [11], i.e. the non zero value of the cross product of the thermal gradient and the gravity vector. So, the observed scaling is the consequence of the balance between the vorticity and the thermal gradient.

In summary, for the convective intensity:

For $R a A^{2} \leq 10$,

$$
\operatorname{Pe} A^{2}=\frac{\pi \sqrt{3}}{432} \operatorname{Ra} A^{2} \approx 1.26 \cdot 10^{-3} \operatorname{Ra} A^{2}
$$

and for $\operatorname{Ra} A^{2} \geq 10^{4}$

$$
\operatorname{Pe} A^{2}=4.6 \cdot 10^{-1}\left(\operatorname{Ra} A^{2}\right)^{2 / 5},
$$

Eq. (6), plotted as a solid line in Figure 3, comes from the asymptotic analysis. In that regime, $P e A^{2}$ is proportional to $R a A^{2}$. Eq. (7) is plotted as a dashed line in Figure 3. The coefficient comes from fitting numerical data to $\left(R a A^{2}\right)^{2 / 5}$, where the $2 / 5$ exponent is explained in [7].

Finally, in order to obtain a practical estimate of the convective intensity, a harmonic average of Eqs. (6) and (7), written as follows, can be used:

$$
P e A^{2}=\left\{\left(1.26 \cdot 10^{-3} R a A^{2}\right)^{-1}+\left[4.6 \cdot 10^{-1}\left(R a A^{2}\right)^{2 / 5}\right]^{-1}\right\}^{-1} .
$$


The dotted-dashed line in Figure 3 represents this solution. It is close to all numerical data with an error smaller than $15 \%$.

\subsection{Nusselt number on the upper limit}

The Nusselt number represents the normalized heat flux. With our normalization, it can be defined locally as follows

$$
N u(x)=\frac{\partial \theta(x, 1)}{\partial y} .
$$

From the asymptotic solution given in Appendix A, the Nusselt number in the conductive regime is given by

$$
\begin{array}{r}
N u(x)=-\frac{\pi^{2} A^{2}}{2}\left(1-A^{2} \frac{\pi^{2}}{3}\right) \cos (\pi A x)+\frac{R a A^{4} \pi^{4} \cos (2 \pi A x)}{1920}- \\
\frac{\left(R a A^{2}\right)^{2} \pi^{4} \cos (\pi A x) \sin ^{2}(\pi A x)}{967680}+\mathcal{O}\left(A^{6},\left(R a A^{2}\right)^{3}\right) .
\end{array}
$$

For small Rayleigh numbers, the leading term of $N u$ is proportional to $A^{2}$. Higher order terms are proportional to powers of $A^{2}$ and $R a A^{2}$. Equation (10) is compared to numerical results on Figure 4. When $R a A^{2}$ is sufficiently small (typically less than $10^{2}$ ), the Nusselt number follows the sinusoidal function as predicted by (10): the local Nusselt number reaches its maximum at the top corners of the enclosure. As $R a A^{2}$ increases, the convective transfer leads to a qualitative change of behavior in the Nusselt number along the longitudinal axis: the local maxima move from the vertical sides to the interior of the enclosure. The asymptotic solution reproduces this trend.

A global expression of the Nusselt number is needed to study the heat flux over the entire range of Rayleigh numbers. Since the average heat flux is null, we take the $L_{2}$ norm of $N u(x)$ defined by:

$$
\langle N u\rangle=\sqrt{A \int_{0}^{1 / A} N u^{2}(x) d x} .
$$

$\langle N u\rangle$, obtained from numerical simulations, is plotted as a function of $R a A^{2}$ in Figure 5. Once again, $R a A^{2}$ appears as a relevant quantity. The results can be summed up as follows:

For $\operatorname{Ra} A^{2} \leq 10$ :

$$
\langle N u\rangle=\frac{\pi^{2} A^{2}}{2 \sqrt{2}}\left(1-A^{2} \frac{\pi^{2}}{3}\right)
$$

and for $\operatorname{Ra} A^{2} \geq 10^{3}$ :

$$
\langle N u\rangle=0.245\left(\operatorname{Ra} A^{2}\right)^{1 / 5} .
$$

Eq. (12) corresponds to the conductive regime represented by horizontal lines in Figure 5. The Nusselt number is independent of $R a A^{2}$ and depends only on the aspect ratio squared. Eq. (13) is a consequence of the scaling law for the convective regime whose the coefficient is a fit of numerical data. In this regime, the heat flux is driven by the boundary layer occurring just below the upper limit of the cavity. 

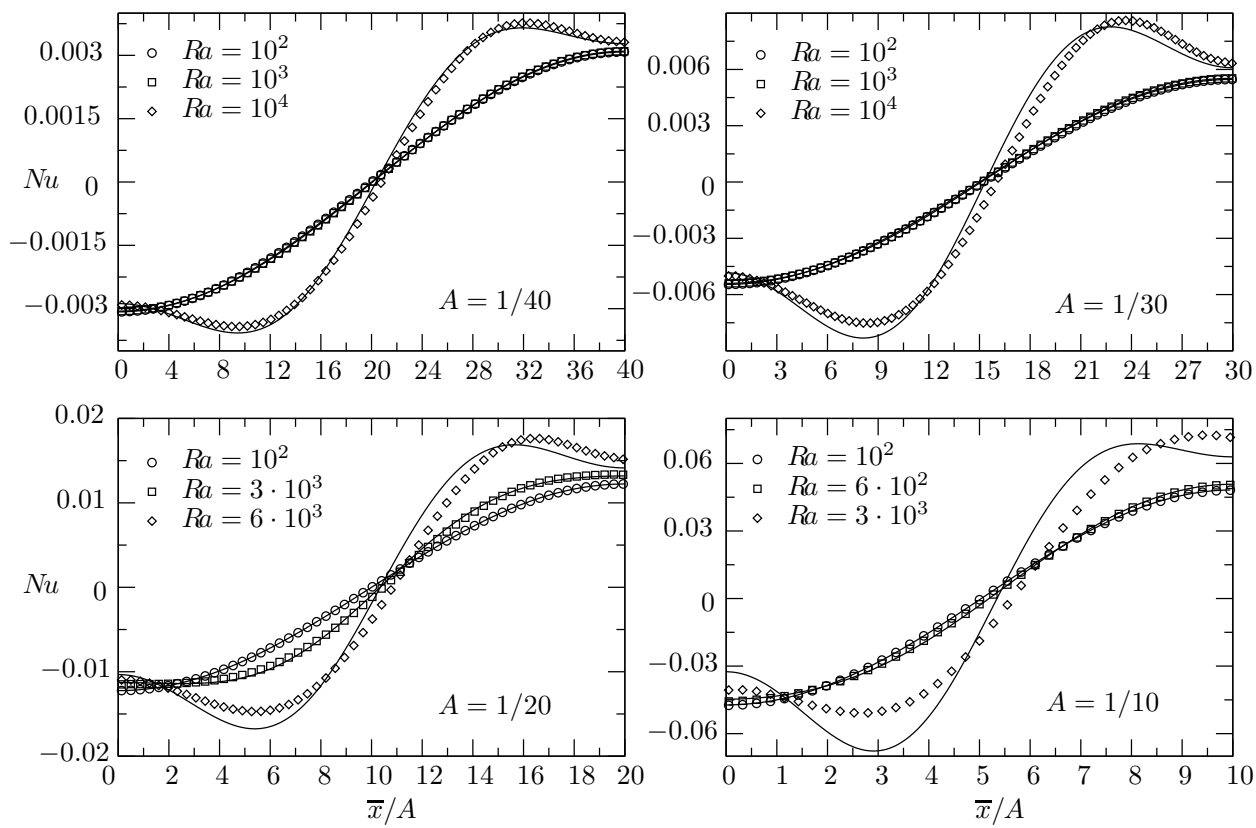

Figure 4: $N u$ as a function of $\bar{x} / A$ for aspect ratios equal to $1 / 40,1 / 30,1 / 20$ and $1 / 10$. Solid lines represent the Nusselt number obtained from Eq. (10).

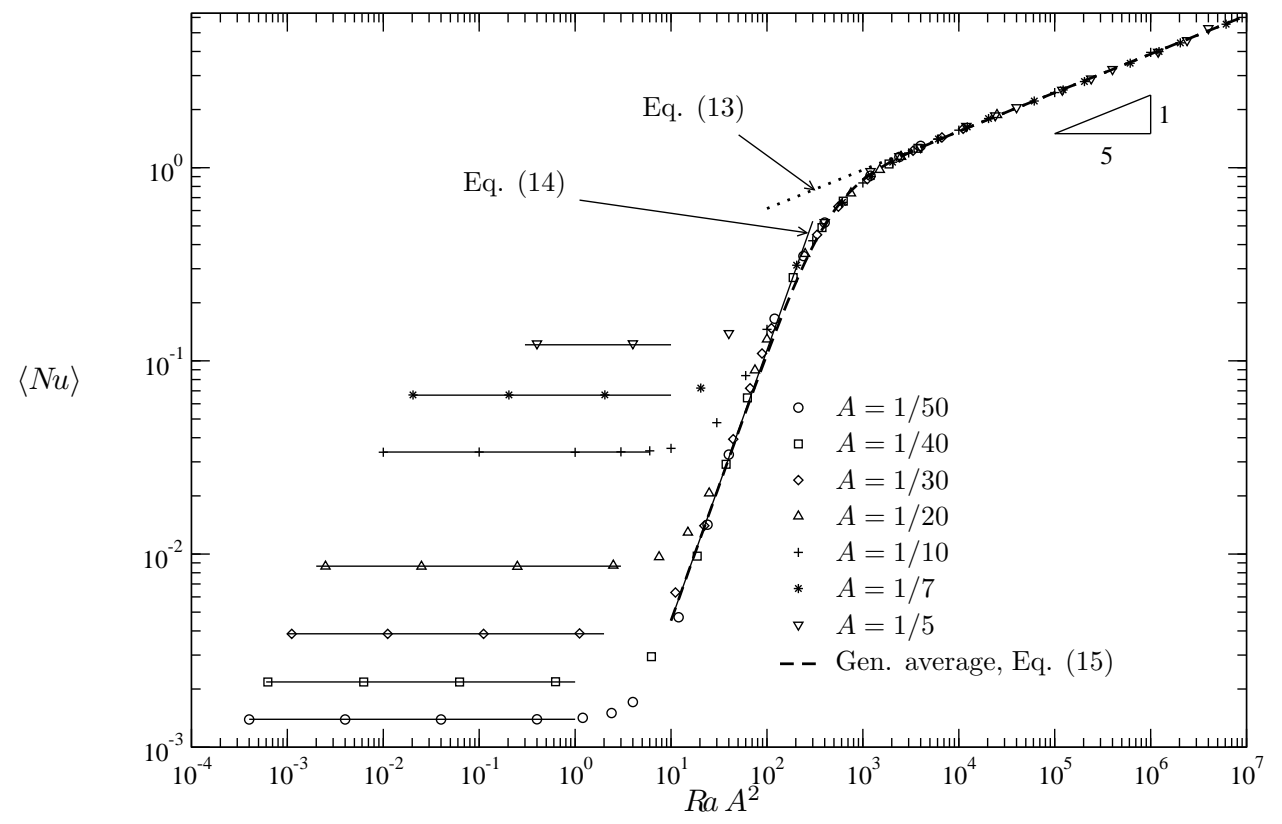

Figure 5: $\langle N u\rangle$ as a function of $R a A^{2}$ for cavities with aspect ratios of $1 / 50,1 / 40,1 / 30,1 / 20,1 / 10,1 / 7$ and $1 / 5$. 
The scaling obtained in the convective regime can be easily recovered using a simple argument. Under the convective regime, cavity height is irrelevant to scale the boundary layer thickness and dimensional analysis shows that the only possible length scale is $\left[\kappa \nu L^{2} /(g \beta \Delta T)\right]^{1 / 5}$. Consequently, the laws for the Nusselt number in the convective regime should hold no matter what temperature profile is applied to the upper boundary of a long cavity filled with a high Prandtl number fluid.

Between these two asymptotic regimes, i.e. for $\left.R a A^{2} \in\right] 10 ; 10^{3}[$, the Nusselt number rises sharply due to the contribution of convection. Heat flux must be a function of $R a A^{2}$ when convection becomes predominant. A fit in this intermediate region is:

$$
\langle N u\rangle=1.8 \cdot 10^{-4}\left(R a A^{2}\right)^{1.4} \text {. }
$$

The approximation (14), represented by solid line in Figure 5, accurately represents the transition regime.

As for convective intensity, a generalized average of (14) and (13) is obtained by

$$
\langle N u\rangle=\left\{\left[1.8 \cdot 10^{-4}\left(R a A^{2}\right)^{1.4}\right]^{-3 / 2}+\left[0.245\left(R a A^{2}\right)^{1 / 5}\right]^{-3 / 2}\right\}^{-2 / 3} .
$$

This gives a practical estimate of the Nusselt number for the transition and convective regimes as is shown by the dashed line in Figure 5.

\subsection{Comparison with previous results}

The results obtained in this article can be compared to previous works. As already pointed out by Böhrer [3], the $R a A^{2}$ parameter is the control parameter even if the temperature is imposed on the upper limit. The agreement is the consequence of the meaning of the quantity $R a A^{2}$. This product can be seen as the ratio of thermal diffusion time over $H$ to the advection time over the length $L$. Low values of $R a A^{2}$ mean that thermal diffusion is efficient. When $R a A^{2}$ is high, thermal advection is the relevant phenomena that controls the thermal field.

In ref. [4], $R a$ is based on the cavity length. So $A^{5} R a$, against which $P e$ is plotted for $A<1$, is equivalent to our expression $R a A^{2}$. Moreover, the Péclet number in [4] is defined as the maximum of the stream function, i.e. the integral of the velocity field. In the conductive regime, the scaling obtained in [4] is equivalent to our result where the Péclet number is proportional to the Rayleigh number. In the convective regime, the Péclet number scales as $\left(R a A^{2}\right)^{1 / 5}$ according to Chiu-Webster et al. whilst our results show that $\mathrm{Pe} A^{2}$ is proportional to $\left(R a A^{2}\right)^{2 / 5}$. The apparent discrepancy is a consequence of the definition of $P e$. Indeed in the convective regime, the derivative along the $y$-axis of the stream function scales like $1 / \delta$ where $\delta$ is the scale of the boundary layer below the upper limit. As pointed out in $[7], \delta \propto 1 /\left(R a A^{2}\right)^{1 / 5}$. Consequently, the expression found in [4] is in agreement with our scaling.

As for the Péclet number, the comparison of the Nusselt number with the previous work of Chiu-Webster et al. [4] has to be carefully studied. In [4], the Nusselt number is taken as the average of the absolute value of the thermal gradient and based on the longitudinal dimension. In the conductive regime, Chiu-Webster et al. show that the Nusselt number is proportional to the aspect ratio whereas our results give a scaling in $A^{2}$. These two results are in fact in perfect agreement if the same spatial scale is used. In the convective regime, our results agree with ones given in [4]. Note that this scaling has been already pointed out by Rossby [14]. 


\section{Conclusion}

In this article, the problem of natural convection in a shallow enclosure is investigated, where a sinusoidal temperature profile is applied to the upper boundary, while the side and bottom walls are adiabatic. With this choice, it is shown that $R a A^{2}$ (see definition in Nomenclature) remains the control parameter. Two main regimes emerge: the first one, observed for $R a A^{2}<10$, is dominated by conduction whereas the second, for $R a A^{2}>$ $10^{3}$, is driven by convection. Analytical laws for convective intensity and heat flux are derived that are based on an analytical asymptotic expansion and on extensive numerical calculations. Under the convective regime, numerical coefficients for these laws come from fitting numerical data, their functional form relying on analytical expansions.

An expression for convective intensity is given over the whole range of $R a A^{2}$ based on a harmonic average. When $R a A^{2}$ belongs to $\left[10 ; 10^{2}\right]$, an approximate relationship is proposed to describe the sharp increase in heat flux. Finally, a generalized average describes the heat flux in both the transition, when $R a A^{2} \in\left[10 ; 10^{2}\right]$, and the convective regimes. The choice of variables makes these expressions directly applicable to practical situations.

From these developments, the characteristic velocity under the conductive regime is shown to be proportional to $R a \kappa / L=\beta g \Delta T H^{3} /(\nu L)$. This parameter is highly sensitive to cavity height and depends also on length and dynamic viscosity but not on heat conductivity. In the convective regime, characteristic velocity is independent of enclosure height since it can be written as $u_{0} \sim(\beta \Delta T g / \nu)^{2 / 5} \kappa^{3 / 5} L^{1 / 5}$ but increases with length and thermal conductivity. Viscosity's importance is reduced since it appears with a $2 / 5$ exponent. These results provide physically grounded answers to the empirical suggestions given by Trier [19].

Regarding heat transfer, the conductive regime leads to a thermal flux on the upper boundary, $\partial T / \partial n$, proportional to enclosure height and inversely proportional to length squared. Under the convective regime, heat flux becomes independent of enclosure height: as with characteristic velocity it is completely driven by the boundary layer below the upper limit.

These results serve as guides to analyze heat and mass transfer in more complex situations closer to those experienced in industrial plants.

\section{A. Asymptotic solution for small aspect ratio}

In the conductive regime, flow develops in the whole cavity. Therefore, spatial length scales are proportional to enclosure size and spatial coordinates are normalized as follows ${ }^{2}$

$$
\begin{aligned}
& \bar{x}=\frac{x}{L}, \\
& \bar{y}=\frac{y}{H} .
\end{aligned}
$$

The results obtained in [7] are used to normalize velocity in the conductive regime.

\footnotetext{
${ }^{2}$ In this appendix, the bar is used over dimensionless variables.
} 
The scaling of $u$ and $v$ is:

$$
\begin{gathered}
\bar{u}=\frac{u}{\kappa R a / L}, \\
\bar{v}=\frac{v}{\kappa R a H / L^{2}} .
\end{gathered}
$$

With this scaling, balance equations are written:

$$
\begin{aligned}
\frac{\partial \bar{u}}{\partial \bar{x}}+\frac{\partial \bar{v}}{\partial \bar{y}} & =0 \\
A^{2} \frac{R a}{\operatorname{Pr}}\left(\bar{u} \frac{\partial \bar{u}}{\partial \bar{x}}+\bar{v} \frac{\partial \bar{u}}{\partial \bar{y}}\right) & =-\frac{\partial \bar{P}}{\partial \bar{x}}+A^{2} \frac{\partial^{2} \bar{u}}{\partial \bar{x}^{2}}+\frac{\partial^{2} \bar{u}}{\partial \bar{y}^{2}} \\
A^{4} \frac{R a}{\operatorname{Pr}}\left(\bar{u} \frac{\partial \bar{v}}{\partial \bar{x}}+\bar{v} \frac{\partial \bar{v}}{\partial \bar{y}}\right) & =-\frac{\partial \bar{P}}{\partial \bar{y}}+A^{4} \frac{\partial^{2} \bar{v}}{\partial \bar{x}^{2}}+A^{2} \frac{\partial^{2} \bar{v}}{\partial \bar{y}^{2}}+\theta \\
\operatorname{Ra} A^{2}\left(\bar{u} \frac{\partial \theta}{\partial \bar{x}}+\bar{v} \frac{\partial \theta}{\partial \bar{y}}\right) & =A^{2} \frac{\partial^{2} \theta}{\partial \bar{x}^{2}}+\frac{\partial^{2} \theta}{\partial \bar{y}^{2}} .
\end{aligned}
$$

Equations (20a-20d) are similar to those used by Bejan [2] (chapter 5) and can be used to find the perturbation solution expanded in terms of $A^{2}$ at small Rayleigh numbers. From equation (20d), the expansion in terms of $A^{2}$ is valid when $R a A^{2} \ll 1$. Note that the problem is singular [6] since all $x$-derivatives disappear if $A$ is taken equal to zero. Actually, the form (20a-20d) corresponds to an external solution valid in the enclosure's core.

To find the asymptotic solution, all quantities are developed as (see Bejan [2])

$$
f=f_{0}+A^{2} f_{1}+A^{4} f_{2}+\cdots+A^{2 i} f_{i}+\cdots .
$$

At zeroth order, the solution is ${ }^{3}$

$$
\begin{aligned}
\bar{u}_{0}(\bar{x}, \bar{y}) & =\pi \sin (\pi \bar{x}) \mathcal{A}_{0}^{\prime}(\bar{y}) \\
\bar{v}_{0}(\bar{x}, \bar{y}) & =-\pi^{2} \cos (\pi \bar{x}) \mathcal{A}_{0}(\bar{y}), \\
\theta_{0}(\bar{x}, \bar{y}) & =\frac{1}{2}[1-\cos (\pi \bar{x})],
\end{aligned}
$$

with

$$
\mathcal{A}_{0}(\bar{y})=\frac{\bar{y}^{2}(\bar{y}-1)^{2}}{48} .
$$

At first order, the solution is

$$
\begin{aligned}
\bar{u}_{1}(\bar{x}, \bar{y}) & =\frac{R a \pi^{3}}{2304} \sin (2 \pi \bar{x})\left[\mathcal{A}_{1}^{\prime}(\bar{y})+\frac{\mathcal{B}_{1}^{\prime}(\bar{y})}{\operatorname{Pr}}\right]+\frac{\pi^{3}}{24} \sin (\pi \bar{x}) \mathcal{C}_{1}^{\prime}(\bar{y}), \\
\bar{v}_{1}(\bar{x}, \bar{y}) & =-\frac{R a \pi^{4}}{1152} \cos (2 \pi \bar{x})\left[\mathcal{A}_{1}(\bar{y})+\frac{\mathcal{B}_{1}(\bar{y})}{\operatorname{Pr}}\right]-\frac{\pi^{4}}{24} \cos (\pi \bar{x}) \mathcal{C}_{1}(\bar{y}), \\
\theta_{1}(\bar{x}, \bar{y}) & =\frac{R a \pi^{2} \sin ^{2}(\pi \bar{x})}{96} \mathcal{D}_{1}(\bar{y})-\frac{\pi^{2}}{4} \cos (\pi \bar{x})\left(\bar{y}^{2}-1\right),
\end{aligned}
$$

\footnotetext{
${ }^{3}$ The symbol ' corresponds to differentiation with respect to $\bar{y}$.
} 
with

$$
\begin{aligned}
& \mathcal{A}_{1}(\bar{y})=\frac{\bar{y}^{9}}{630}-\frac{\bar{y}^{8}}{140}+\frac{\bar{y}^{7}}{105}-\frac{\bar{y}^{4}}{30}+\frac{16 \bar{y}^{3}}{315}-\frac{3 \bar{y}^{2}}{140}, \\
& \mathcal{B}_{1}(\bar{y})=-\frac{\bar{y}^{9}}{252}+\frac{\bar{y}^{8}}{56}-\frac{\bar{y}^{7}}{30}+\frac{\bar{y}^{6}}{30}-\frac{\bar{y}^{5}}{60}+\frac{\bar{y}^{3}}{252}-\frac{\bar{y}^{2}}{840} \\
& \mathcal{C}_{1}(\bar{y})=\frac{\bar{y}^{6}}{20}-\frac{\bar{y}^{5}}{10}-\frac{\bar{y}^{4}}{6}+\frac{13 \bar{y}^{3}}{30}-\frac{13 \bar{y}^{2}}{60}, \\
& \mathcal{D}_{1}(\bar{y})=\frac{\bar{y}^{5}}{5}-\frac{\bar{y}^{4}}{2}+\frac{\bar{y}^{3}}{3}-\frac{1}{30} .
\end{aligned}
$$

At zeroth order, the temperature is similar to the boundary condition on $\bar{y}=1$. The function $\mathcal{A}_{0}(\bar{y})$ is identical to the one obtained by Cormack et al. [5]. Function $\bar{u}_{0}$ differs from the Cormack et al. solution by the quantity $\pi \sin (\pi \bar{x}) / 2$. The velocity solution at zeroth order is derived from the purely conductive temperature field. The function $\bar{u}_{0}$ is minimum and maximum for $\bar{y}$ equal to $(3+\sqrt{3}) / 6$ and $(3-\sqrt{3}) / 6$, respectively. At first order, the thermal solution has two terms: the first linked to convection and the second to conductive transfer.

To determine the local Nusselt number in the conductive regime, the integral form of energy equation (20d) is used. Indeed, integration over the vertical direction with boundary conditions gives the local Nusselt number:

$$
N u(\bar{x})=-A^{2} \frac{d^{2}}{d \bar{x}^{2}} \int_{0}^{1} \theta(\bar{x}, \bar{y}) d \bar{y}+\operatorname{Ra} A^{2} \frac{d}{d \bar{x}} \int_{0}^{1} \bar{u} \theta d \bar{y} .
$$

By taking the two first orders of the asymptotic solution, equation (10) is found. The integro-differential equation (23) is useful to determine the local Nusselt number since the term proportional to $A^{4}$ is obtained without the solution at second order.

\section{References}

[1] G. K. Batchelor. Heat tranfer by free convection across a closed cavity between vertical boundaries at different temperatures. Quart. Appl. Math., 12:209-233, 1954.

[2] A. Bejan. Convection heat transfer. John Wiley \& Sons, New York, 1995.

[3] B. Böhrer. Convection in a long cavity with differentially heated end walls. Int. J. Heat Mass Transfer, 40:4105-4114, 1997.

[4] S. Chiu-Webster, E. J. Hinch, and J. R. Lister. Very viscous horizontal convection. J. Fluid Mech., 611:395-426, 2008.

[5] D. E. Cormack, L. G. Leal, and J. Imberger. Natural convection in a shallow cavity differentially heated end walls. Part 1. Asymptotic theory. J. Fluid Mech., 65:209-229, 1974.

[6] M. Van Dyke. Perturbations methods in fluid mechanics. The parabolic Press, Stanford, California, 1975.

[7] J.-M. Flesselles and F. Pigeonneau. Kinematic regimes of convection at hight Prandtl number in a shallow cavity. C. R. Mécanique, 332:783-788, 2004.

[8] C. A. J. Fletcher. Computational Techniques for Fluid Dynamics. Volume II: Specific Techniques for Different Flow Categories. Springer-Verlag, Berlin, 1991.

[9] R. Gardon. A review of radiant heat transfer in glass. J. Am. Ceram. Soc., 44:305-312, 1961.

[10] H. J. J. Gramberg, P. D. Howell, and J. R. Ockendon. Convection by a horizontal thermal gradient. J. Fluid Mech., 586:41-57, 2007.

[11] D. D. Joseph. Stability of fluid motions II. Springer-Verlag, Berlin, 1976.

$[12]$ B. P. Leonard. A stable and accurate convective modelling procedure based on quadratic upstream interpolation. Comput. Methods Appl. Mech. Engrg., 19(1):59-98, 1979. 
[13] K.-O. Lim, T.-H. Song, and K.-S. Lee. Patterns of natural convection driven by the free surface temperature distribution in a glass melting furnace. Glass Technol., 39:27-31, 1998.

[14] H. T. Rossby. On thermal convection driven by non-uniform heating from below: an experimental study. Deep-Sea Res., 12:9-16, 1965.

[15] H. T. Rossby. Numerical experiments with a fluid heated non-uniformly from below. Tellus, 50A:242-257, 1998

[16] H. Scholze. Glass. Nature, Structures and Properties. Springer-Verlag, Berlin, 1990.

[17] R. Siegel and J. Howell. Thermal Radiation Heat Transfer. Taylor \& Francis, Levitown, 2002.

[18] R. C. J. Somerville. A nonlinear spectral model of convection in a fluid unevenly heated from below. J. Atmos. Sci., 24:665-676, 1967.

[19] W. Trier. Glass furnaces, design construction and operation. Society of glass technology, Sheffield, 1984. 